\title{
Adaptive Instrument Mlodule: Space Instrument Controller "Brain" through Programmable Logic Devices
}

\author{
Ann Garrison Darrin \\ The Johns Hopkins Lniversity Applied Physics \\ Laboratory \\ Richard Conde \\ The Johns Hopkins Lniversity Applied Physics \\ Laboratory \\ Bobbie Chern (intern) \\ The Johns Hopkins University Applied Physics \\ Laboratory
}

\author{
Phil Luers \\ V.t.St Goddard Space Flighr c'enter
}

Steve Jurczyk

NASA Langley Research center

Carl Mills

NASA Langley Research Center

\begin{abstract}
The Adaptive Instrument Module (AlM) will be the first true demonstration of reconfigurable computing with field-programmable gate arras (FPGAs) in space. enabling the "brain" of the ststem to evolve or adapt to changing requirements. In partnership with N.ASA Goddard Space Flight Center and the Australian Cooperative Research Centre for Satellite Systems (CRCSS). APL has built the flight version to be flow'n on the Australian university-class satellite FEDSAT.

The All provides satellites the flexibility to adapt to changing mission requirements by reconfiguring standartized processing hurdware rather than incurring the large costs associated with new builds. This ability to reconfigure the processing in response to changing mission needs leads to true evolveable computing. wherein the instrument "brain" can learn from new science data in order to perform state-of-the-art data processing. The development of the $A 1 \mathrm{H}$ is significant in its enormous potential to reduce total life-cicle costs for fiature space exploration missions. The advent of RALbased FPGits whose configuration can be changed at any time has enabled the development of the $A / \mathrm{l} / \mathrm{for}$ processing tasks that condd not be performed in sofirare. The use of the Alll shahles reconfiguration of the FPG.t circutry while the spacesut is in flight. with moms acompaning advantages. The HII demonstrates the practicalities of asing reconfigurable computing hardware devices hy conducting a series of designed experiments. These inclade the demonstration of implemsang dats compression data filterits and
\end{abstract}

communication message processing and inter-experiment data computation. The second generation is the Adaptive Processing Template (ADAPT) which is further described in this paper.

The next step forward is to make the hardware itself adaptable and the ADAPT parsues this challenge by developing a reconfigurable module that will be capable of functioning efficiently in various applications. ADAPT will take advantage of radiation tolerant R.A-based field programmable gate arrav (FPG.4) technolog: to develop a reconfigurable processor that combines the flexibility of a general purpose processor running sofware with the performance of application specific processing hardura for a variety of high performance computing applications.

The Adaptive Instrument Module (AIM) will be the first true demonstration of reconfigurable computing with field-programmable gate arrays (FPGAs) in space. enabling the "brain" of the system to evolve or adapt to changing requirements. In partnership with N.ASA Goddard Space Flight Center and the Australian Cooperative Research Centre for Satellite Systems (CRCSS). APL has built the tlight version to be flown on the Australian university-class satellite FEDSAT

The AIM provides satellites the flexibility to adapt to changing mission requirements by reconfiguring standardized processing hardware rather than incurring the large costs associated with new builds. This ability to recontigure the processing in response to changing mission needs leads to true evolveable computing. wherein the instrument "brain" can learn from new 
science data in order to perform state-of-the-dit data processing. The development of the MM is significan in its enormous potential to reduce total life-cycle costs for future space exploration missions. The advent of RAMbased PPGAs whose contiguration can be changed at any time has enabled the development of the AIM for processing tasks that could not be performed in software. The use of the AlM enables recontiguration of the FPGA circuitry while the spacecraft is in flight. with many accompanying advantages. The AIM demonstrates the practicalities of using reconfigurable computing hardware devices bs conducting a series of designed experiments. These include the demonstration of implementing data compression, data filtering, and communication message processing and inter-experiment data computation. The second generation is the Adaptive Processing Template (ADAPT) which is further described in this paper.

\section{Adaptive Processing Template (ADAPT)}

In the past, system flexibility was primarily associated with system software. New functions and applications were accomplished by changing the sequence of instructions executed by a general-purpose processor. The next step forward is to make the hardware itself adaptable and the ADAPT pursues this challenge by developing a reconfigurable module that will be capable of tunctioning efficiently in various applications. ADAPT will take advantage of radiation tolerant RAM-based field programmable gate array (FPGA) technology to develop a reconfigurable processor that combines the flexibility of a general purpose processor running software with the performance of application specific processing hardware for a variety of high performance computing applications. This improved performance along with the adaptability of this technology provides significant benefits at several levels:

1. Although the basic physical hardware design remains unchanged. the hardware for the control and data interfaces can easily be programmed for a specific instrument andor spacecraft data system architecture This allows flight qualification of the basic hardware independent of the detailed hardware design for a specific system. This minimizes the cost of developing the basic hardware for each instrument and allows the physical design to be easily reprogrammed for any system architecture.

2. This approach also minimizes overall instrument or ststem development time by allowing physical and electrical testing of the hardware to proceed concurrently with the detailed design and programming of the FPGAs. Also. it an error in the design is detected during system level testing. the hardware can be easily reconfigured without having to physically remove the processor from the ststem. This minmises the impact on the project schedule and risk to the tlight hardware.

3. The use of RAM-based FPGA technology enables the hardware design to be recontigured in flight to overcome both hardware and software errors that may be detected after launch during mission operations. This reduces overall mission risk which is becoming more important as light system development times and budgets decrease in the current faster. cheaper, and better environment.

4. The ability to reconfigure the ADAPF in tlight allows the processor functions to adapt to changing mission conditions and also allows improved on board processing algorithms to be uploaded to the instrument. The reconfigured processor can optimize mission operations to exploit science "targets of opportunity" as the mission progresses and also take advantage of improved data processing algorithms to return the most science for the minimum cost.

5 Reconfigurable hardware provides another level of fault tolerance. The hardware can be reprogrammed in flight to replace the function of faulty circuit components that might otherwise cause the instrument to fail. If some part of the FPGA fails, the circuitry can be redesigned and the hardware reprogrammed to avoid the faulty portions of the component. This essentially provides inherent redundancy to improve the overall mission probability of success with none of the traditional costs associated with redundant processors.

6. The use of multiple FPGAs in a processor provides a scalable architecture. The hardware can be configured as a parallel processor by sending the data to multiple FPGAs simultaneously or configured as a pipeline processor by sending the data to one FPGA first and then a second FPGA to complete the necessary processing. This allows the architecture to be optimized for the specific data processing algorithms required for an instrument providing improved performance without redesigning the basic physical hardware for each application.

An Advanced Technology Development (ATD) project at JHU APL is developing a standalone reconfigurable logic ASIC Simulator board that has many similarities to the ADAPT. This board stores configurations in flash memory, and allows new configurations to be downloaded by a host processor over an RS-232 link. It uses a Kilinx Virtex FPGA. the same type planned for use in the ADAPT. A photo of the module is shown in Figure 1. and a block diagram in figure? 

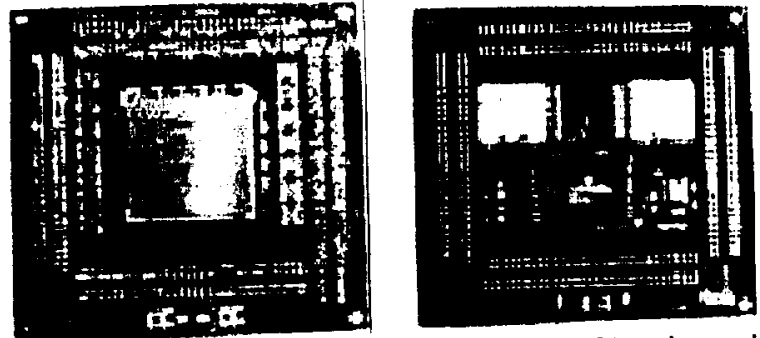

Figure 1. Reconfigurable Logic/ASIC Simulator based on Virtex FPGA

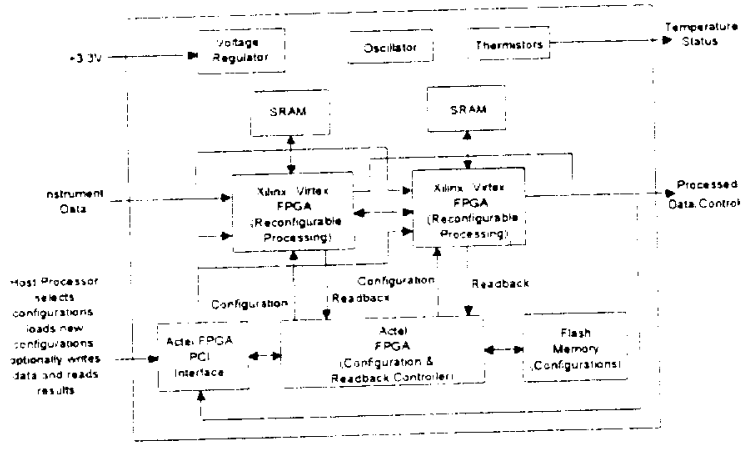

Figure 2. ADAPT System Hardware Architecture

\section{ADAPT Hardware Design}

The ADAPT implements complex algorithms directly in reconfigurable hardware for processing of high rate instrument data. A diagram of the ADAPT hardware architecture is shown in Figure 3. Two state of the art Xilinx Virtex FPGAs are the heart of the ADAPT. Each contains the equivalent of 1.1 million gates and 131,000 bits of RAM. A wide range of cores are available for these FPGAs, including DSP functions, processors, and math functions. New designs can be implemented with a wide range of development tools. In the ADAPT design. each Virtex FPGA is connected to SRAM memory to implement additional storage for intermediate results, coefficients, and variables that some algorithms may require. The Virtex FPGAs are interconnected to allow data to pass between them. Each is connected to the instrument data input. and each can output processed data or control information. Having at least two FPGAs on the board also provides some redundancy in case of device failure.

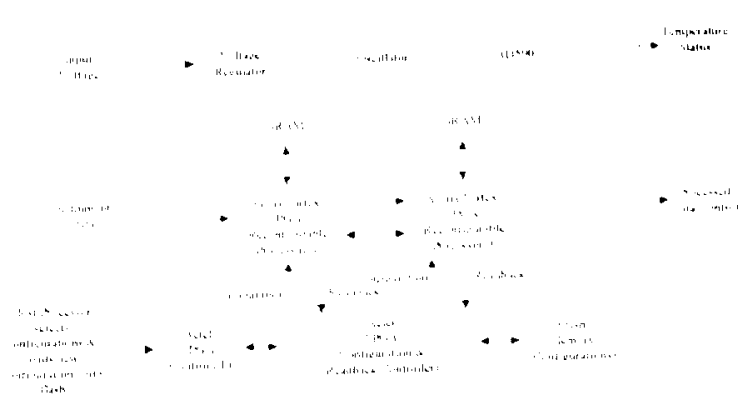

Figure 3. ADAPT Hardware Architecture

The ADAPT stores multiple FPGA contigurations in flash memory. The host processor selects which configurations are to be used. This allows the instrument data processing algorithms to be changed in realtime. A fuse-programmed Actel FPGA implements the system backplane $\mathrm{PCl}$ interface to allow the system host processor to select the configurations to be loaded into the Virtex FPGAs. A second Actel includes the circuitry to read back the configuration from the Xilinx FPGAs and compare it to the original configuration in flash memory. If any discrepancies are detected. the configuration is autonomously reloaded, and the host processor is notified that data processing will be halted for a few seconds, Instrument data may either inputted either through the $\mathrm{PCl}$ bus or an $\mathrm{L} / \mathrm{O}$ connector. Processed data may be either outputted over the $\mathrm{PCl}$ bus or an $\mathrm{I} / \mathrm{O}$ connector.

The ADAPT includes a voltage regulator to supply power to the Virtex FPGAs. The voltage regulator is kept on the ADAPT board so that the host system will not have to generate $+2.5 \mathrm{~V}$. It is expected that the card will only need to be supplied with $+3.3 \mathrm{~V}$ from the backplane. An oscillator supplies the clock needed to run the Actel FPGAs. The temperature of the Virtex parts and the linear regulator will be measured by thermistors. The thermistors will be connected to an $\mathrm{l} / \mathrm{O}$ connector.

\section{Spacecraft Architecture with ADAPT}

An instrument that uses the ADAPT card can either have its own Compact PCl chassis, or it could be integrated directly ino the spacecraft Compact $\mathrm{PCl}$ chassis. The second approach would vield a lower mass system. since the digital electronics for the instrument the ADAPT card) could exist as an additional card in the spacecraft chassis, rather than as a standalone chassis. An example of this architecture is shown in figure 4. Since the bulk of the instrument processing is done in the ADAPT card. the spacecraft processor card can do the remainder of the processing with a small percentage of its resources, for example pachetizing the reduced science data or 
transferring the packets to the spatcectaft solid state recorder. If the extral mass is availatice a separate Instrument Compact PCI chassis with a dedicated processor could be implemented.

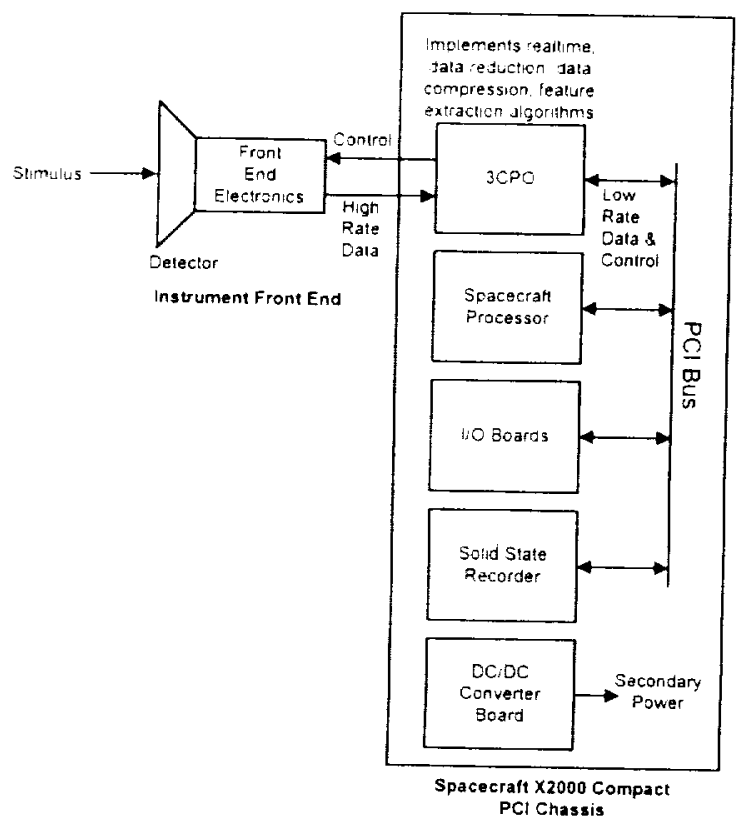

Figure 4. Spacecraft and Instrument Architecture with ADAPT

\section{Technology Description and Benefits}

In the past, system flexibility was primarily associated with system software. New functions and applications were accomplished by changing the sequence of instructions executed by a general-purpose processor. The next step forward is to make the hardware itself adaptable and the ADAPT pursues this challenge by developing a reconfigurable processor board that will be capable of functioning efficiently in various applications. ADAPT will take advantage of radiation tolerant RAMbased field programmable gate array (FPGA) technology to develop a reconfigurable processor that combines the tlexibility of a general purpose processor running software with the performance of application specific processing hardware for a variety of high performance computing applications.

\section{Potential Science Application}

One of the most challenging aspects of planetary spectroscopy is identifying previously unknown sites which have a sought after mineralogic signature. measuring them. and downlinking the data. The simplest method, measuring and downlinking data that cover the entirety of a surface of interest. is appropriate for a limited volume of data having relatively low spatial resolution. However it is impractical for high spatial resolution data dice to the comequenty wery high data volume. A somewhat more sophisticated approach would be to acyuire a large volume of data. analyze it onboard, and downlink only selected portions. However this method too fails at very high spatial resolutions

ADAPT would provide an alternative implementation that is tar more efficient in usage of spacecraft resources: the AD АP Would allow an instrument to acquire and discard data continually in a standby mode. analyzing it "on the fly" and deciding autonomously when a valuable data segment should be saved. For example. taking as an example the Martian case, as few as 14 chamels could be analyzed continuously ( 3 parameterizing ferric iron absorption bands at 660 and $860 \mathrm{~nm}$. 3 parameterizing the $2000-\mathrm{nm}$ atmospheric $\mathrm{CO}_{2}$ band, 3 parameterizing the $2350-\mathrm{nm}$ carbonate band. and 2 parameterizing the 3000 $\mathrm{nm} \mathrm{H}_{2} \mathrm{O}$ band). These absorptions are diagnostic of $\mathrm{key}$ aqueous phases. Key parameters for data calibration and processing would be stored in the ADAPT, including offset and gain corrections for dark current and radiometric sensitivity and a model atmospheric spectrum. The selected wavelengths from data acquired continuously in "standby" mode would be calibrated on the fly, and a first-order atmospheric correction would be performed by scaling the model atmospheric spectrum using measured $2000-\mathrm{nm} \mathrm{CO}_{2}$ band depth. The processed spectra would then be parameterized and thresholded to determine when one of the mineralogic absorptions exceeded a critical value indicative of interesting mineralogy. At that point an autonomous decision would be made whether to begin a predetermined sequence of appropriate instrument operations and data storage. Similar analysis of spectra being saved would determine whether continued acquisition of new data warranted the overwriting of an existing data segment saved previously. via the same decision making process.

These algorithms will be designed for implementation in the Xilinx FPGA on the ADAPT. They will be simulated on a Mentor Graphics workstation, and simulated spectrometer data will be used as the stimulus. This will validate the design and ability of the ADAPT to meet the SSE goals of reducing downlink and operation autonomously. The planned increase in information extraction for a given downlink is 1000.1. The reduction in time to resume mission operations after mission interruption due to engineering anomaly as well as engineering setup time for science observations. should be reduced to the time to select and load one of the prestored FPGA configurations on the ADAPT. Once commanded to a new configuration. the ADAPT should load it in well under 10 seconds. 
(1) Bergman. $N$ and Dawood. A. Recontigurable Computers in Space: Problems, Solutions and Future Directions Dagstuhl Seminar 0)(1261

[2] Bezerra . E.A A Distributed Fault-Tolerant System Bised on Reconfigurable Computing Technology for Space Instrument Processing.CNPq process: 200008,98-6 Space Sciance Centre. School of Engineering and Information Technology Lniversity of Sussex. June 2000

[3] Conde, R.. Dumont, C. and Darrin. A.G.-"Adaptive Instrument Module through Programmable Logic , 1999 Military and Aerospace Applications of Programmable Devices and Technologies Conference, Laurel, MD,

September 28-30 1999

[4] Flatley, T.P. Developing Reconfigurable Computing Systems for Space Flight Applications, 1999 Military and Aerospace Applications of Programmable Devices and Technologies Conference. Laurel. MD, September 28-30 1999

The Adaptive Data Analysis and Processing Technology (ADAPT) project is a joint JHU/APL. Langley Research Center project and the Goddard Space Flight Center. 
